\title{
Improved Carbonylation of Heterocyclic Chlorides and Challenging Aryl Bromides
}

\author{
Jennifer Albaneze-Walker*, Charles Bazaral, Tanya \\ Leavey, Peter G. Dormer, and Jerry A. Murry
}

Department of Process Research, Merck \& Co., Inc., P. O. Box 2000, Rahway, NJ 07065-0900 USA

I. General Information $\quad$ S-1

II. General Carbonylation Procedure $\quad$ S-1

III. Heterocyclic Chlorides to Methyl Esters $\quad$ S-2

III. Electron-Rich Aryl Bromides to Methyl Esters $\quad$ S-4

\section{General}

General Methods. ${ }^{1} \mathrm{H}$ NMR and ${ }^{13} \mathrm{C}$ NMR were recorded at ambient temperature on a Bruker DPX 400 at a frequency of 400.13 and $100.61 \mathrm{MHz}$, respectively. The chemical shifts are reported in ppm relative to residual $\mathrm{CHCl}_{3}(\delta=7.27)$ for proton and $\mathrm{CDCl}_{3}(\delta=77.0)$ for carbon. The data are reported as follows: proton multiplicities $(\mathrm{s}=$ singlet, $\mathrm{d}=$ doublet, $\mathrm{t}=$ triplet, $\mathrm{q}=$ quartet, $\mathrm{m}=$ multiplet, $\mathrm{br}=$ broad, and app = apparent), coupling constants, and integration. Microanalyses were performed by Quantitative Technologies, Inc. Melting points are reported uncorrected. Flash chromatography was performed using the indicated solvent system on EM Reagents silica gel $\left(\mathrm{SiO}_{2}\right) 60$ (230-400 mesh).

Materials. $\mathrm{MeOH}$ and triethylamine were used as received. All reagents used were commercially available from Aldrich Chemical Co.

\section{General Procedure for Carbonylation of Heterocyclic Chlorides and Aryl Bromides to Esters}

An autoclave vessel was charged with chloride or bromide substrate ( $3 \mathrm{mmol}$ ), (BINAP)PdCl $24 \mathrm{mg}$ (1 $\mathrm{mol} \%)$, and triethylamine $0.9 \mathrm{~mL}(1.3 \mathrm{eq})$ in $15 \mathrm{~mL}$ of methanol. Vessel was tested for leaks using nitrogen, then purged with nitrogen three times and carbon monoxide three times. Vessel was pressurized to 50 psig with carbon monoxide and heated to $100^{\circ} \mathrm{C}$. The reaction was thus allowed to progress until completion, then allowed to cool to room temp and sampled. Reaction was judged to be complete when $2 \%$ LCAP or less of starting material remained. When cool, the reaction solution was filtered through Solka-Floc, and cake rinsed with methanol, and filtrate concentrated, and purified by silica gel chromatography to provide the products listed in Table 2 and Table 3.

\section{Heterocyclic Chlorides to Esters}


The known chloride substrates in Table 2: 2-chloropyridine, 2,5-dichloropyridine, 2,3-dichloropyridine, 3chloropyridine, 3-chloro-2-fluoro-5-trifluoromethylpyridine, 2-chloropyrazine, and 1-chloroanthraquinone were commercially available from Sigma-Aldrich. Entry 8 substrate naphthyridone chloride was synthesized. ${ }^{1}$

\section{Representative Procedure for the Carbonylation of Chlorides to Esters (Table 2). 2-Chloropyridine.}<smiles>COC(=O)c1ccccn1</smiles>

An autoclave vessel was charged with 2-chloropyridine 340mg (3 mmol), (BINAP) $\mathrm{PdCl}_{2} 24 \mathrm{mg}$ (1 mol\%), and triethylamine $0.9 \mathrm{~mL}(1.3 \mathrm{eq})$ in $15 \mathrm{~mL}$ of methanol. Vessel was tested for leaks using nitrogen, then purged with nitrogen three times and carbon monoxide three times. Vessel was pressurized to $50 \mathrm{psig}$ with carbon monoxide and heated to $100^{\circ} \mathrm{C}$. The reaction was thus allowed to progress until completion, then allowed to cool to room temp and sampled. Reaction was judged to be complete when 2\%LCAP or less of starting material remained. When cool, the reaction solution was filtered through Solka-Floc, and cake rinsed with methanol, and filtrate concentrated. Assay yield of methyl picolinate was determined by HPLC analysis of the reaction mixture referenced to authentic (Sigma-Aldrich) methyl picolinate (407 mg, $99 \%)$.

\section{Dimethyl 2,5-dicarboxypyridine. ${ }^{2}$}

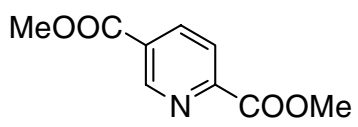

Using the general procedure with 2,5-dichloropyridine (444 mg, $3 \mathrm{mmol}$ ) afforded dimethyl 2,5-

dicarboxypyridine, after purification by flash chromatography (10\% EtOAc in hexanes), as a white solid (573 mg, 98\%): ${ }^{1} \mathrm{H}$ NMR $\left(\mathrm{CDCl}_{3}, 400 \mathrm{MHz}\right) \delta 3.99(\mathrm{~s}, 3 \mathrm{H}), 4.04(\mathrm{~s}, 3 \mathrm{H}), 8.20(\mathrm{dd}, J=8.2,0.8 \mathrm{~Hz}, 1 \mathrm{H}), 8.42$ $(\mathrm{dd}, J=8.2,2.1 \mathrm{~Hz}, 1 \mathrm{H}), 9.30(\mathrm{dd}, J=2.0,0.7 \mathrm{~Hz}, 1 \mathrm{H}) .{ }^{13} \mathrm{C}$ NMR $\delta$ 49.0, 49.5, 120.9, 124.9, 134.6, 147.0, 147.1, 161.1, 161.2.

\section{Methyl 3-chloro-2-carboxypyridine. ${ }^{3}$}

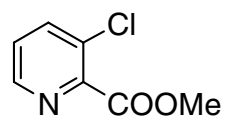

Using the general procedure with 2,3-dichloropyridine (444 mg, $3 \mathrm{mmol}$ ) afforded methyl 3-chloro-2carboxypyridine, after purification by flash chromatography (10\% EtOAc in hexanes), as a white solid (443 mg, 86\%): ${ }^{1} \mathrm{H} \mathrm{NMR}\left(\mathrm{CDCl}_{3}, 400 \mathrm{MHz}\right) \delta 3.88(\mathrm{~s}, 3 \mathrm{H}), 7.28$ (dd, $\left.J=8.1,4.7 \mathrm{~Hz}, 1 \mathrm{H}\right), 7.69$ (dd, $J=8.1$, $1.5 \mathrm{~Hz}, 1 \mathrm{H}), 8.44(\mathrm{dd}, J=4.7,1.4 \mathrm{~Hz}, 1 \mathrm{H}) .{ }^{13} \mathrm{C}$ NMR $\delta 52.8,126.3,130.9,138.6,147.1,147.15,164.5$.

Carbonylation of 2-fluoro-3-chloro-5-(trifluoromethyl)pyridine. Using the general procedure with 2fluoro-3-chloro-5-(trifluoromethyl)pyridine $(600 \mathrm{mg}, 3 \mathrm{mmol})$ afforded two products, separated by flash chromatography (5-15\% EtOAc in hexanes): Methyl 2-methoxy-5-(trifluoromethyl)nicotinate.<smiles>COC(=O)c1c[14c](C(F)(F)F)cnc1OC</smiles>

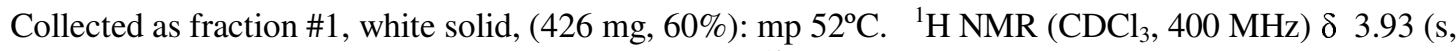
$3 \mathrm{H}), 4.10(\mathrm{~s}, 3 \mathrm{H}), 8.37,(\mathrm{~d}, J=2.7,1 \mathrm{H}), 8.57(\mathrm{~m}, 1 \mathrm{H}) .{ }^{13} \mathrm{C}$ NMR $\delta 52.5,54.8,113.8,119.5\left(\mathrm{q}, J_{\mathrm{CF}}=271.5\right.$

\footnotetext{
${ }^{1}$ Springfield, S.; Marcantonio, K.; Ceglia, S.; Albaneze-Walker, J.; Dormer, P.; Nelson, T.; Murry J. J. Org. Chem. 2003, 68, 4598-4599.

2 Jokela, R. Magn. Res Chem. 1992, 30, 681-683.

${ }^{3}$ Epsztajn, J.; Plotka, M.; Grabowska, A. Synth. Comm. 1997, 27, 1075-1086.
} 
$\mathrm{Hz}), 123.0\left(\mathrm{q}, J_{\mathrm{CF}}=33.6 \mathrm{~Hz}\right), 138.4,148.0,164.0(\mathrm{C}=\mathrm{O})$. Anal Calcd for $\mathrm{C}_{9} \mathrm{H}_{8} \mathrm{~F}_{3} \mathrm{NO}_{3}: \mathrm{C}, 45.97 ; \mathrm{H}, 3.43 ; \mathrm{F}$, 24.24; N, 5.96. Found: C, 45.92; H, 3.11; F, 24.57; N, 5.86. Anal Calcd for $\mathrm{C}_{9} \mathrm{H}_{8} \mathrm{~F}_{3} \mathrm{NO}_{3}$ : C, 45.97; H, 3.43; F, 24.24; N, 5.96. Found: C, 45.92; H, 3.11; F, 24.57; N, 5.86.

Dimethyl 5-(trifluoromethyl)pyridine-2,3-dicarboxylate.<smiles>COC(=O)c1cc(C(F)(F)F)cnc1C(=O)OC</smiles>

Collected as fraction \#2, colorless oil (79 mg, 10\%): ${ }^{1} \mathrm{H}$ NMR $\delta 3.97$ (s, 3H), $4.01(\mathrm{~s}, 3 \mathrm{H}), 8.44(\mathrm{~s}, 1 \mathrm{H})$, $9.00(\mathrm{~s}, 1 \mathrm{H}) .{ }^{13} \mathrm{C}$ NMR $\delta 53.20,53.24,122.4$ (q, $\left.J_{\mathrm{CF}}=273.1 \mathrm{~Hz}\right), 126.5,127.7$ (q, $\left.J_{\mathrm{CF}}=34.4 \mathrm{~Hz}\right), 135.1$, 148.6, 153.9, $164.0(\mathrm{C}=\mathrm{O}), 165.6(\mathrm{C}=\mathrm{O})$. Anal Calcd for $\mathrm{C}_{10} \mathrm{H}_{8} \mathrm{~F}_{3} \mathrm{NO}_{4}: \mathrm{C}, 45.64 ; \mathrm{H}, 3.06 ; \mathrm{F}, 21.66 ; \mathrm{N}$, 5.32. Found: C, 45.45; H, 2.86; F, 21.80; N, 5.29.

Methyl carboxypyrazine. ${ }^{4}$

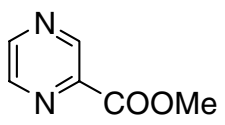

Using the general procedure with chloropyrazine (344 $\mathrm{mg}, 3 \mathrm{mmol}$ ) afforded methyl carboxypyrazine, after purification by flash chromatography (5\% EtOAc in hexanes), as a white solid (410 mg, 99\%): ${ }^{1} \mathrm{H}$ $\operatorname{NMR}\left(\mathrm{CDCl}_{3}, 400 \mathrm{MHz}\right) \delta 4.04(\mathrm{~s}, 3 \mathrm{H}), 8.72(\mathrm{dd}, J=2.4,1.7 \mathrm{~Hz}, 1 \mathrm{H}), 8.77$ (d, J=2.4 Hz, 1H), 9.31 (d, $J=1.5 \mathrm{~Hz}, 1 \mathrm{H}) .{ }^{13} \mathrm{C}$ NMR $\delta 53.0,143.2,144.3,146.2,147.6,164.3$.

\section{Methyl 1-carboxyanthraquinone. ${ }^{5}$}<smiles>COC(=O)c1cccc2c1C(=O)c1ccccc1C2=O</smiles>

Using the general procedure with 1-chloroanthraquinone (728 mg, $3 \mathrm{mmol}$ ) afforded methyl 1carboxyanthraquinone [32114-46-8], after purification by flash chromatography (5\% EtOAc in hexanes), as a white solid (487 mg, 61\%): ${ }^{1} \mathrm{H} \mathrm{NMR}\left(\mathrm{CDCl}_{3}, 400 \mathrm{MHz}\right) \delta 4.02(\mathrm{~s}, 3 \mathrm{H}), 7.83-7.86(\mathrm{~m}, 2 \mathrm{H})$, 8.32-8.37 (m, 2H), $8.40(\mathrm{~d}, J=8.2 \mathrm{~Hz}, 1 \mathrm{H}), 8 .(\mathrm{dd}, J=8.44,1.6 \mathrm{~Hz}, 1 \mathrm{H}), 8.96(\mathrm{~d}, J=1.8 \mathrm{~Hz}, 1 \mathrm{H}) .{ }^{13} \mathrm{C} \mathrm{NMR} \delta$ $52.7,127.2$, 127.3, 127.4, 128.5, 133.2, 133.3, 133.4, 134.3, 134.4, 134.5, 135.0, 136.0, 165.4, 182.1, 182.4 .

6-Ethyl-2-methyl-8-cyclopropyl-2-fluoro-5-oxo-5,8-dihydro-1,8-naphthyridine-2,6-dicarboxylate. ${ }^{1}$<smiles>CCOC(=O)c1cn(C2CC2)c2nc(C(=O)OC)c(F)cc2c1=O</smiles>

Using the general procedure with 6-Ethyl-2-chloro-8-cyclopropyl-2-fluoro-5-oxo-5,8-dihydro-1,8naphthyridine-6-carboxylate $(932 \mathrm{mg}, 3 \mathrm{mmol})$ afforded 6-ethyl-2-methyl-8-cyclopropyl-2-fluoro-5oxo-5,8-dihydro-1,8-naphthyridine-2,6-dicarboxylate, after purification by flash chromatography $(20 \%$ EtOAc in hexanes), as a white solid, $(632 \mathrm{mg}, 63 \%): \mathrm{mp} 226^{\circ} \mathrm{C} .{ }^{1} \mathrm{H} \mathrm{NMR} \delta 1.07(\mathrm{~m}, 2 \mathrm{H}), 1.32(\mathrm{~m}, 2 \mathrm{H})$, $1.41(\mathrm{t}, J=7.1 \mathrm{~Hz}, 3 \mathrm{H}), 3.75(\mathrm{sept}, J=3.9 \mathrm{~Hz}, 1 \mathrm{H}), 4.06(\mathrm{~s}, 3 \mathrm{H}), 4.41(\mathrm{q}, J=7.1 \mathrm{~Hz}, 2 \mathrm{H}), 8.52\left(\mathrm{~d}, J_{\mathrm{HF}}=9.1 \mathrm{~Hz}\right.$, $1 \mathrm{H}), 8.73(\mathrm{~s}, 1 \mathrm{H}) .{ }^{13} \mathrm{C}$ NMR $\delta 7.6,14.3,34.3,53.2,61.1,111.5,124.5\left(\mathrm{~d}, J_{\mathrm{CF}}=21.6 \mathrm{~Hz}\right), 126.5,139.6(\mathrm{~d}$, $\left.J_{\mathrm{CF}}=14.4 \mathrm{~Hz}\right), 145.9,149.6,156.1\left(\mathrm{~d}, J_{\mathrm{CF}}=267.5 \mathrm{~Hz}\right), 162.7,164.7,173.1$. Anal Calcd for $\mathrm{C}_{16} \mathrm{H}_{15} \mathrm{FN}_{2} \mathrm{O}_{5}: \mathrm{C}_{\text {, }}$ 57.49; H, 4.52; F, 5.68; N, 8.38. Found: C, 56.99; H, 4.34; F, 5.90; N, 8.22.

\footnotetext{
${ }^{4}$ Takeuchi, R.; Suzuki, K.; Sato, N. Synthesis, 1990, 923-4.

${ }^{5}$ Gore, P.; Rahim, A.; Waters, D. J. Chem. Soc. (B) 1971, 202-204.
} 


\section{Aryl Bromides to Esters}

Representative Procedure for the the Carbonylation of Bromides to Esters (Table 3). Methyl 2-aminobenzoate. ${ }^{6}$<smiles>COC(=O)c1ccccc1N</smiles>

An autoclave vessel was charged with 2-bromoaniline $172 \mathrm{mg}$ (1 mmol), (BINAP) $\mathrm{PdCl}_{2} 24 \mathrm{mg}$ (3 mol\%), and triethylamine $0.42 \mathrm{~mL}(1.3 \mathrm{eq})$ in $7 \mathrm{~mL}$ of methanol. Vessel was tested for leaks using nitrogen, then purged with nitrogen three times and carbon monoxide three times. Vessel was pressurized to 50 psig with carbon monoxide and heated to $100^{\circ} \mathrm{C}$. The reaction was thus allowed to progress until completion, then allowed to cool to room temp and sampled. Reaction was judged to be complete when $2 \%$ LCAP or less of starting material remained. When cool, the reaction solution was filtered through Solka-Floc, and cake rinsed with methanol, and filtrate concentrated to afford methyl 2-aminobenzoate, after purification by flash chromatography (10\% EtOAc in hexanes), as a colorless oil (134 mg, 92\%): ${ }^{1} \mathrm{H} \mathrm{NMR}\left(\mathrm{CDCl}_{3}, 400\right.$ MHz) $\delta 3.88(\mathrm{~s}, 3 \mathrm{H}), 5.50$ (br s, 2H), 6.63-6.69 (m, 2H), 7.25-7.30 (m, 1H), 7.86 (dd, J=8.0, 1.6, 1H). ${ }^{13}$ C NMR $\delta 51.4,110.8,116.3,116.7,131.1,134.0,150.2,168.5$.

Methyl 3-aminobenzoate. ${ }^{7}$<smiles>COC(=O)c1cccc(N)c1</smiles>

Using the general procedure with 3-bromoaniline $(172 \mathrm{mg}, 1 \mathrm{mmol})$ afforded methyl 3-aminobenzoate, after purification by flash chromatography (25\% EtOAc in hexanes), as a white solid (148 $\mathrm{mg}, 98 \%)$ : ${ }^{1} \mathrm{H}$ $\operatorname{NMR}\left(\mathrm{CDCl}_{3}, 400 \mathrm{MHz}\right) \delta 3.55(\mathrm{br} \mathrm{s}, 2 \mathrm{H}), 3.90(\mathrm{~s}, 3 \mathrm{H}), 6.87(\mathrm{ddd}, J=8.0,2.4,0.8,1 \mathrm{H}), 7.21(\mathrm{t}, J=7.8,1 \mathrm{H})$, $7.36(\mathrm{t}, J=2.1,1 \mathrm{H}), 7.43(\mathrm{dt}, J=8.1,09 ., 1 \mathrm{H}) .{ }^{13} \mathrm{C}$ NMR $\delta 52.0,115.8,119.4,119.7,129.2,131.0,146.2$, 167.2 .

\section{Methyl 4-aminobenzoate. ${ }^{6}$}<smiles>COC(=O)c1ccc(N)cc1</smiles>

Assay yield of methyl 4-aminobenzoate was determined by HPLC analysis of the reaction mixture referenced to authentic (Acros) methyl 4-aminobenzoate ( $76 \mathrm{mg}, 50 \%$ ).

\section{Methyl 4-methoxybenzoate. ${ }^{7}$}

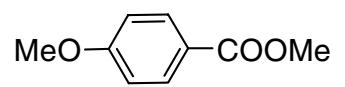

Using the general procedure with 4-bromoanisole (172 $\mathrm{mg}, 1 \mathrm{mmol}$ ) afforded methyl 4methoxybenzoate, after purification by flash chromatography (3\% EtOAc in hexanes), as a white solid $(160 \mathrm{mg}, 96 \%):{ }^{1} \mathrm{H}$ NMR $\left(\mathrm{CDCl}_{3}, 400 \mathrm{MHz}\right) \delta 3.83(\mathrm{~s}, 3 \mathrm{H}), 3.87(\mathrm{~s}, 3 \mathrm{H}), 6.89(\mathrm{~d}, J=9.0,2 \mathrm{H}), 7.98(\mathrm{~d}$, $J=8.9,2 \mathrm{H}) .{ }^{13} \mathrm{C}$ NMR $\delta 51.7,55.3,113.5,122.5,131.5,163.2,166.7$.

\section{Methyl 2-methoxybenzoate. ${ }^{6}$}<smiles>COc1ccccc1C(C)=O</smiles>

Using the general procedure with 2-bromoanisole (172 $\mathrm{mg}, 1 \mathrm{mmol})$ afforded methyl 2methoxybenzoate, after purification by flash chromatography (5\% EtOAc in hexanes), as a white solid

\footnotetext{
${ }^{6}$ The Aldrich Library of ${ }^{13} \mathrm{C}$ and ${ }^{1}$ H FT-NMR Spectra; Aldrich Chemical Company, Inc.: Milwaukee, 1993 ; Ed 1.

${ }^{7}$ Budesinsky, M.; Exner, O. Magn. Res Chem. 1989, 27, 585-591.
} 
(164 mg, 99\%): ${ }^{1} \mathrm{H}$ NMR ( $\left.\mathrm{CDCl}_{3}, 400 \mathrm{MHz}\right) \delta 3.89$ (s, 3H), 3.09 (s, 3H), 6.96-7.00 (m, 2H), 7.44-7.49 (m, $1 \mathrm{H}), 7.79(\mathrm{dd}, J=8.0,1.6,1 \mathrm{H}) .{ }^{13} \mathrm{C}$ NMR $\delta 51.9,55.9,111.9,119.9,120.0,131.6,133.4,159.0,166.6$. 